\title{
Minireview
}

\section{The protein folding challenge in psychrophiles: facts and current issues}

\author{
Florence Piette, ${ }^{\dagger}$ Caroline Struvay ${ }^{\dagger}$ and \\ Georges Feller* \\ Laboratory of Biochemistry, Center for Protein \\ Engineering, University of Liège, Institute of Chemistry \\ B6a, 4000 Liège-Sart Tilman, Belgium.
}

\section{Summary}

The protein folding process in psychrophiles is impaired by low temperature, which exerts several physicochemical constraints, such as a decrease in the folding rate, reduced molecular diffusion rates and increased solvent viscosity, which interfere with conformational sampling. Furthermore, folding assistance is required at various folding steps according to the protein size. Recent studies in the field have provided contrasting and sometimes contradictory results, although protein folding generally appears as a rate-limiting step for the growth of psychrophiles. It is proposed here that these discrepancies reflect the diverse adaptive strategies adopted by psychrophiles in order to allow efficient protein folding at low temperature. Cold adaptations apparently superimpose on pre-existing cellular organization, resulting in different adaptive strategies. In addition, microbial lifestyle further modulates the properties of the chaperone machinery, which possibly explains the occurrence of cold-adapted and non-cold-adapted protein chaperones in psychrophiles.

\section{Introduction}

Psychrophiles (literally, cold-loving organisms) are mainly microorganisms thriving in permanently cold environments and even at subzero temperatures in supercooled liquid water. Such extremely cold conditions are encountered, for instance, in salty cryopegs at $-10^{\circ} \mathrm{C}$ in the permafrost (Gilichinsky et al., 2005) or in the brine veins between polar sea ice crystals at $-20^{\circ} \mathrm{C}$ (Deming, 2002).

Received 18 November, 2010; accepted 12 January, 2011. *For correspondence. E-mail gfeller@ulg.ac.be; Tel. (+32) 436633 43; Fax (+32) 43663364 . 'Equally contributing authors.
Unusual microbiotopes have also been described such as porous rocks in Antarctic dry valleys hosting microbial communities surviving at $-60^{\circ} \mathrm{C}$ (Friedmann, 1982; Cary et al., 2010). These organisms do not merely survive or endure such extremely inhospitable conditions but are irreversibly adapted to these environments, as most psychrophiles are unable to grow at mild (or mesophilic) temperatures. It is frequently overlooked that the majority $(>80 \%)$ of the Earth's biosphere is cold and permanently exposed to temperatures below $5^{\circ} \mathrm{C}$ (Rodrigues and Tiedje, 2008). Such a low mean temperature mainly arises from the fact that $70 \%$ of the Earth's surface is covered by oceans that have a constant temperature of $4^{\circ} \mathrm{C}$ below $1000 \mathrm{~m}$ depth, irrespective of the latitude. The polar regions account for another $15 \%$, to which the glacier and alpine regions must be added, as well as the permafrost representing more than $20 \%$ of terrestrial soils (Cowan et al., 2007; Margesin et al., 2008). All these low temperature biotopes have been successfully colonized by cold-adapted organisms, which include a large range of representatives from all three domains: Bacteria, Archaea and Eukarya. As a result, psychrophiles are the most abundant extremophiles in terms of biomass, diversity and distribution.

Life in cold environments requires a vast array of adaptive features at nearly all levels of the cell architecture and function. Indeed, cold exerts severe physicochemical constraints on living organisms including increased water viscosity, decreased molecular diffusion rates, reduced biochemical reaction rates, perturbation of weak interactions driving molecular recognition and interaction, strengthening of hydrogen bonds that, for instance, stabilize inhibitory nucleic acid structures, increased solubility of gases and stability of toxic metabolites as well as reduced fluidity of cellular membranes (D'Amico et al., 2006; Gerday and Glansdorff, 2007; Margesin et al., 2008; Rodrigues and Tiedje, 2008). Earlier studies of psychrophiles at the molecular level were mainly focused on cold-active enzymes and on maintenance of membrane fluidity because both processes were regarded as prerequisites to the environmental adaptation. It was shown that the high level of specific activity at low temperatures of cold-adapted enzymes is a key adaptation to compensate 
for the exponential decrease in chemical reaction rates as the temperature is reduced. Such high biocatalytic activity arises from the disappearance of various non-covalent stabilizing interactions, resulting in an improved flexibility of the enzyme conformation (Feller and Gerday, 2003; Siddiqui and Cavicchioli, 2006; Feller, 2010). It should be noted that this adaptive feature is genetically encoded within the protein sequence and results from a long-term adaptation. Whereas membrane structures are rigidified in cold conditions, an adequate fluidity is required to preserve the integrity of their physiological functions. This homeoviscosity is achieved by steric hindrances introduced into the lipid bilayer via incorporation of cisunsaturated and branched-chain lipids, a decrease in average chain length, and an increase both in methyl branching and in the ratio of anteiso- to iso-branching (Russell, 2007). This adaptation involves the regulation of pre-existing biosynthetic pathways. More recently, several genomes from psychrophilic bacteria and Archaea have been sequenced (Casanueva et al., 2010) but only a few of these have been analysed with respect to cold adaptation (Saunders et al., 2003; Rabus et al., 2004; Medigue et al., 2005; Methe et al., 2005; Duchaud et al., 2007; Riley et al., 2008; Rodrigues et al., 2008; Allen et al., 2009; Ayala-del-Rio et al., 2010). Proteomic and transcriptomic studies of cold-adapted microorganisms have also been used to search for cellular functions that are stimulated for growth in the cold (Goodchild et al., 2004; 2005; Qiu et al., 2006; Bakermans et al., 2007; Kawamoto et al., 2007; Zheng et al., 2007; Bergholz et al., 2009; Campanaro et al., 2011; Ting et al., 2010; Williams et al., 2010).

For many years, protein synthesis and protein folding have been considered as temperature-sensitive cellular processes that severely restrict microbial growth at low temperature in the absence of specific adaptations. Despite this well-recognized limitation, the challenge of protein synthesis and folding in psychrophiles has been addressed only recently, mainly via genomics and proteomics. The various 'omics' technologies (Casanueva et al., 2010) have produced a huge amount of data. This has, however, precluded a fine analysis of the protein folding problem, which is central to microbial cold adaptation. We hope to provide here a comprehensive and integrated survey of this topic (i.e. the facts), in order to highlight some current issues and to suggest future avenues for study in the field.

\section{Protein synthesis in psychrophiles}

Microbial protein synthesis is one of the most complex cellular processes and is subjected to nearly all the above mentioned physicochemical constraints imposed by low temperature. Secondary structures in RNA species are stabilized via $\mathrm{H}$-bond strengthening (Cartier et al., 2010). Availability of the numerous partners (mRNA, tRNA, initiation factors, elongation factors, GTP, etc.) is affected by reduced molecular diffusion rates and by increased cytoplasmic viscosity. Recognition and docking of these partners with ribosomes is altered by the opposite temperature dependence of the weak interactions involved (electrostatic interactions are stabilized by a decrease in temperature whereas the hydrophobic effect is weakened). Last but not least, the synthetic activity of ribosomes is decreased, as in almost all biochemical reactions.

At the structural level, very few insights for protein synthesis adaptation in psychrophiles have been reported. The prevalence of $\mathrm{A}: \mathrm{U}$ base pairing in rRNA of psychrophilic prokaryotes, which is less stable than the triplehydrogen bonded $\mathrm{G}: \mathrm{C}$ base pair, has been involved in the improvement of the folded ribosome function in cold-adapted microorganisms (Galtier and Lobry, 1997; Khachane et al., 2005). Unprecedented high levels of post-transcriptional modification of tRNA by dihydrouridine have been reported in three psychrophilic bacteria (Dalluge et al., 1997). The specific chemical properties of dihydrouridine perturb the stacking that usually stabilizes RNA molecules and it has been proposed that dihydrouridine confers local structural flexibility to tRNA molecules. In addition, RNA helicases have been found to be overexpressed at low temperature in many psychrophilic microorganisms such as Methanococcoides burtonii (Lim et al., 2000), Exiguobacterium sibiricum (Rodrigues et al., 2008), Sphingopyxis alaskensis (Ting et al., 2010), Psychrobacter arcticus (Zheng et al., 2007; Bergholz et al., 2009) and Pseudoalteromonas haloplanktis (Piette et al., 2010). These helicases may help to unwind the RNA secondary structures for efficient translation in the cold (Cartier et al., 2010). At the genome level, an interesting observation is the relatively high number of rRNA genes and of tRNA genes (up to 106 genes, sometimes organized in long runs of repeated sequences), at least in $P$. haloplanktis (Medigue et al., 2005), Colwellia psychrerythraea (Methe et al., 2005) and Psychromonas ingrahamii (Riley et al., 2008). This could reflect the need for a high capacity for translation in the cold, but this is by no means a general trait of psychrophilic microorganisms. Nevertheless, these examples indicate that psychrophiles have indeed evolved adaptive mechanisms to optimize protein synthesis.

Proteomic and transcriptomic studies have compared the relative abundance of protein synthesized at low $\left(-6^{\circ} \mathrm{C}\right.$ to $\left.4^{\circ} \mathrm{C}\right)$ and high $\left(18^{\circ} \mathrm{C}\right.$ and higher) culture temperatures but they have also provided a contrasted pattern on this topic. In the case of $P$. haloplanktis for instance, $30 \%$ of the upregulated proteins at $4^{\circ} \mathrm{C}$ were found to be 
directly related to protein synthesis (Piette et al., 2010). It was concluded that protein synthesis may be a ratelimiting step for growth in the cold, therefore inducing a cellular response. A similar pattern is indeed observed in several cold-adapted bacteria, in particular S. alaskensis (Ting et al., 2010), which overexpress proteic components of both transcription and translation. However, in many cases, the proportion of these proteins in the upregulated proteome is less significant and precludes a generalization of the conclusions made for $P$. haloplanktis. In the archaeon $M$. burtonii, a notable feature is the upregulation of genes involved in maintaining RNA in a state suitable for translation and for enabling translation initiation (Campanaro et al., 2011).

\section{Protein folding in the cold}

Following its linear synthesis on the ribosome, a polypeptide has to adopt its final and biologically active three-dimensional conformation. The forces driving protein folding are essentially the same as those driving the formation of oil micelles in water: the entropic cost of encaging non-polar groups in the water molecule network is high and the system evolves towards the burial of these groups within a globular structure, away from the water molecules in the solvent. This hydrophobic effect is a primary determinant of protein folding. During folding, the process is further modulated by interactions between groups that have been brought into contact. In proteins, van der Walls interactions and $\mathrm{H}$-bonds are the most abundant but salt bridges, aromatic interactions, formation of disulfide bonds and prolyl isomerization (see below) also make substantial contributions, as well as secondary structures and various scaffolds (Jenkins and Pickersgill, 2001) that are prone to adaptive modifications.

The rate of protein folding is adversely affected by low temperature because this corresponds to the rate of a reaction starting from the unstructured nascent polypeptide and ending in the folded native state. Folding also requires some level of conformational sampling which is disfavoured by the reduction in diffusion rates and by the increased solvent viscosity, according to the Kramers theory (Kramers, 1940). More intriguingly, the hydrophobic effect proceeds endothermically and is therefore weakened by a decrease in temperature. Furthermore, the hydrophobic core of psychrophilic proteins tends to be reduced and these proteins frequently expose a high fraction of non-polar residues at the solvent accessible surface: both parameters disfavour a compact conformation by weakening the hydrophobic effect on folding (Smalas et al., 2000; Feller and Gerday, 2003; Siddiqui and Cavicchioli, 2006; Feller, 2010). Taken together, these aspects strongly suggest that the main folding

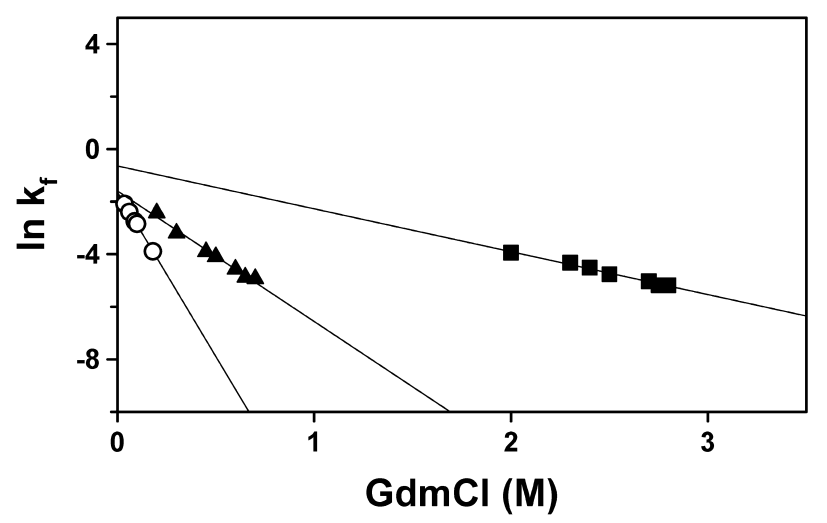

Fig. 1. Determination of intrinsic folding rate constants, $k_{\mathrm{f}}$, for homologous psychrophilic (open circles), mesophilic (triangles) and hyperthermophilic (squares) proteins. Convergence of extrapolations at $0 \mathrm{M}$ guanidinium chloride $(\mathrm{GdmCl})$ concentration indicates similar intrinsic folding rates (see text for details).

driving force is perturbed in psychrophiles. Accordingly, there is an obvious need for biophysical studies on protein folding in psychrophiles, which are currently lacking.

Ongoing research in our laboratory has provided initial insights into the basic aspects in this field. Figure 1 illustrates the determination of the folding rate constants for three homologous proteins from psychrophilic, mesophilic and hyperthermophilic bacteria. Kinetics were recorded (here by fluorescence) starting with the fully unfolded protein in a denaturant, guanidinium chloride, then by diluting the denaturant down to a final residual concentration indicated on the $x$-axis. The range of denaturant concentrations used for each protein correlates with its stability: for instance, higher concentrations are required to unfold the most stable protein. The intrinsic folding rate constants, $k_{\mathrm{f}}$, are calculated by extrapolation of the experimental values to a nil denaturant concentration on the $y$-axis. As shown in Fig. 1, the intrinsic rate constants of the three proteins are similar within the experimental error, despite huge differences in stability. Therefore, at a given temperature, these proteins fold at the same rate and there is no evidence that their structure has been subjected to adaptive adjustments allowing different folding rates. Accordingly, the hyperthermophilic protein should fold extremely fast at its environmental temperature $\left(\sim 85^{\circ} \mathrm{C}\right)$ but the psychrophilic protein should fold very slowly at near-zero temperatures. This raises at least two questions: first, is a slow folding rate detrimental to psychrophilic microorganisms?; second, is folding assistance of prime importance for cold adapted polypeptides? While the first question remains unanswered, the upregulation of protein chaperones in psychrophilic proteomes indicates their significant contribution in adaptive mechanisms. 


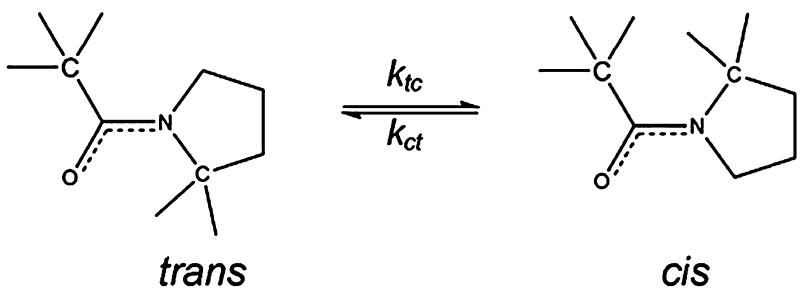

Fig. 2. Trans and cis isomers of a peptidyl-prolyl peptide bond. Reprinted with permission from Zoldak and colleagues (2009). Copyright 2009 American Chemical Society.

\section{Peptidyl-prolyl cis/trans isomerization}

The trans conformation of the peptide bond is favoured for all amino acids because of the steric hindrances imposed by the side-chains of adjacent residues. Such steric constraint is less relevant for the cyclic side-chain of proline, bound to the amino group, and the cis conformation is energetically less unfavourable. Furthermore, the cistrans isomerization of the peptidyl-prolyl bond (Fig. 2) is an intrinsically slow reaction, because it involves the rotation about the peptide bond, which has a partial doublebond character (Baldwin, 2008). As a result, proline isomerization is a rate limiting step for the folding of most proteins but living cells are equipped with catalysts, prolyl isomerases (PPiases or rotamases), which accelerate the isomerization process (Fig. 3). These specialized chaperone catalysts exist as individual proteins or as catalytic domains in a larger molecule, such as the trigger factor (see below). Referring back to psychrophilic proteins, these slow-folding polypeptides are further impaired in the acquisition of their final conformation by proline isomerization. Moreover, the spontaneous rate of the proline

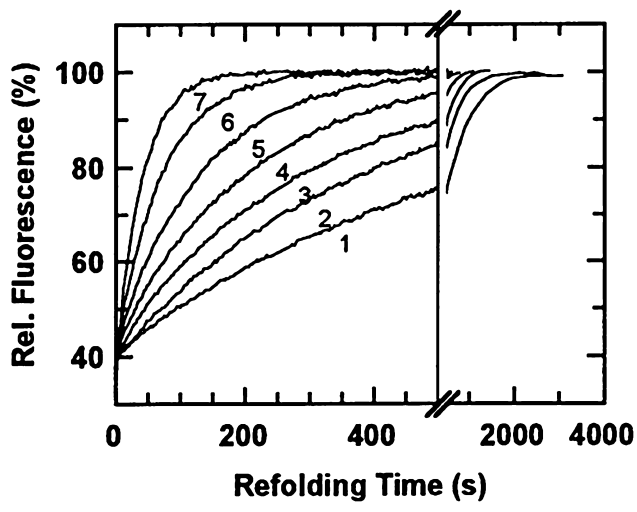

Fig. 3. Catalytic activity of PPiases on protein folding. The refolding rate of protein limited by proline isomerization can be slow (1). Addition of increased amounts of PPiase (2-7) accelerates the folding rate. Reprinted by permission from Macmillan Publishers: The EMBO Journal (Scholz et al., 1997), copyright 1997.

isomerization reaction is slowed down at low temperature. How do psychrophilic microorganisms cope with proline isomerization?

In order to improve molecular flexibility, many coldadapted proteins tend to possess a reduced proline content (Feller and Gerday, 2003; Siddiqui and Cavicchioli, 2006; Feller, 2010) that should attenuate the negative effect of proline isomerization on folding. But very significantly, PPiases are overexpressed at low temperature in the proteome of most psychrophilic bacteria analysed so far (Table 1), and sometimes at very high levels. This involves cytoplasmic PPiases from the cyclophilin family in $P$. arcticus, M. burtonii and PPiD in P. haloplanktis (a cyclophilin-type PPiase implicated in the folding of membrane proteins), a parvulin-type PPiase in

Table 1. Upregulated chaperones (+) and downregulated chaperones (-) in psychrophilic microorganisms grown at low temperature.

\begin{tabular}{|c|c|c|c|c|c|c|c|}
\hline & P. articus ${ }^{\mathrm{a}}$ & $\begin{array}{l}\text { Shewanella SIB1 }{ }^{\mathrm{b}} \\
\text { S. livingstonensis }\end{array}$ & M. burtonif & S. alaskensis ${ }^{\mathrm{e}}$ & P. haloplanktis ${ }^{f}$ & E. sibiricum ${ }^{g}$ & $\begin{array}{l}\text { E. sibiricum } \\
\text { transcriptome }^{\text {h }}\end{array}$ \\
\hline PPiase & $+/-$ & + & + & + & + & & \\
\hline GroEL & + & & - & + & - & & - \\
\hline GroES & + & & & + & & & - \\
\hline DnaK & & & - & & - & + & - \\
\hline DnaJ & - & & - & $+/-$ & & & - \\
\hline TF & - & + & & & + & + & \\
\hline ClpB & + & & + & + & & & \\
\hline $\mathrm{sH}$ sp & & & + & - & - & & \\
\hline Hsp90 & & & & & - & & \\
\hline Dsb & + & & & & - & & \\
\hline
\end{tabular}

a. Zheng et al. (2007); Bergholz et al. (2009).

b. Suzuki et al. (2004).

c. Kawamoto et al. (2007).

d. Goodchild et al. (2004; 2005); Campanaro et al. (2011).

e. Ting et al. (2010).

f. Piette et al. (2010) and F. Piette, S. D’Amico, G. Mazzucchelli, A. Danchin, P. Leprince and G. Feller, unpublished results.

g. Qiu et al. (2006).

h. Rodrigues et al. (2008).

$(+/-)$ corresponds to proteins from the same family that are differently regulated.

(C) 2011 Society for Applied Microbiology and Blackwell Publishing Ltd, Environmental Microbiology, 13, 1924-1933 


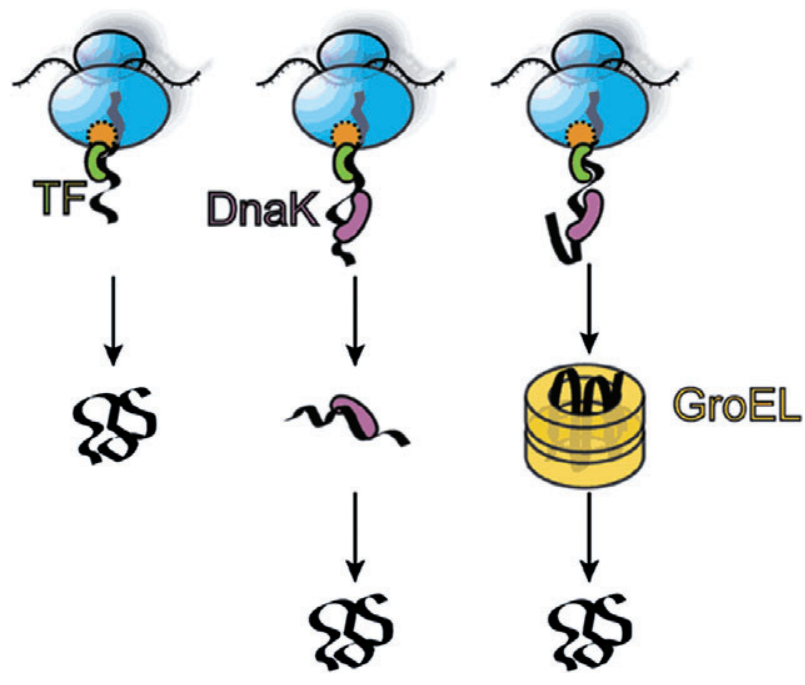

Fig. 4. In bacteria, TF interacts with emerging nascent chains at the ribosomal exit site. Some polypeptides then interact with the bacterial chaperones DnaK and GroEL, which assist the folding of selected subsets of cellular proteins. Reprinted by permission from Macmillan Publishers: Nature Structural Biology (Albanese and Frydman, 2002), copyright 2002.

S. alaskensis, the FKBP-type PPiases in M. burtonii and Schewanella SIB1, as well as the trigger factor, a ribosome-bound chaperone possessing a PPiase domain (Stoller et al., 1995), in Shewanella livingstonensis (Kawamoto et al., 2007), E. sibiricum (Qiu et al., 2006) and $P$. haloplanktis (Piette et al., 2010).

Such recurrent and consistent observations also strongly suggest that protein folding is a rate-limiting step for psychrophiles, which induces a cellular response aimed at facilitating and accelerating the slowest event in the acquisition of the biologically active conformation of proteins. It should be mentioned that $P$. arcticus upregulates a cyclophilin-type PPiase at low temperature but also upregulates an FKBP-type PPiase at high temperature: this is a first insight into the coexistence of gene sets, sharing similar function, but involved in protein folding at opposite environmental temperatures.

\section{Folding assistance by protein chaperones}

Although some proteins fold spontaneously in vitro, most of them require in vivo assistance by molecular chaperones to facilitate folding to the native state in the crowded cytosol (for a review, see Hartl and Hayer-Hartl, 2009). In bacteria, chaperone-assisted folding of newly synthesized polypeptides involves three main partners (Fig. 4). The ribosome-bound trigger factor (TF) is the first chaperone interacting co-translationally with virtually all nascent polypeptides, and most small proteins ( $70 \%$ of total) may fold rapidly upon synthesis without further assistance. Longer chains ( $20 \%$ of total) interact subsequently with
DnaK (and its co-chaperones DnaJ, GrpE) and fold upon cycles of ATP-dependent binding and release. About 10\% of polypeptides transit the ATP-dependent chaperonin system GroEL/ES, which functions by enclosing proteins in a cage-like nanocompartment. The latter is essential (gene deletion is lethal in bacteria) because a number of important proteins are crucially chaperonin-dependent for folding. It should be mentioned that these chaperones do not speed up the folding process but rather optimize the efficiency of folding by avoiding aggregation and misfolding. Other accessory chaperones are also involved such as Hsp90, the small sHsp like IbpA and B, the disaggregation chaperone $\mathrm{ClpB}$ or Dsb proteins for disulfide bond formation (Table 1).

Not surprisingly, the genome of psychrophiles contains a full set of genes coding for the chaperone machinery. But this is in contrast with hyperthermophilic Archaea which, unexpectedly, only possess minimal protein folding systems (Laksanalamai et al., 2004), although it has been anticipated that high temperatures would require efficient folding assistance. Finally, it is worth mentioning that the trigger factor is a cold shock protein in Escherichia coli (Kandror and Goldberg, 1997) whereas the other chaperones are well-known heat shock proteins (HSPs). This significant distinction appears to be of prime importance for psychrophiles.

\section{Differential expression of chaperones at low temperature}

The differential expression of protein chaperones in psychrophiles was first highlighted in $P$. haloplanktis (Piette et al., 2010) because of the well-contrasted pattern observed in this strain. Indeed, this Antarctic bacterium strongly overexpresses the trigger factor (TF) at low temperature ( 40-fold) whereas it represses most other HSP chaperones to nearly undetectable levels (Fig.5). Upregulation of TF has been also observed in S. livingstonensis and E. sibiricum, and the transcriptome of the latter strain has revealed a similar pattern of HSP downregulation (Table 1). Such imbalance in the chaperone machinery has several implications.

i. Downregulation of HSP chaperones severely impairs an essential cellular function as these chaperones assist co- or post-translational protein folding and prevent or relieve misfolding. Therefore, it appears that the ribosome-bound TF, which interacts with virtually all nascent polypeptides, is essential to initiate proper folding in these psychrophiles. Incidentally, low temperature is well known to reduce the probability of misfolding and aggregation (King et al., 1996), therefore possibly reducing the need for HSP chaperones that act downstream from TF. 
$4^{\circ} \mathrm{C}$
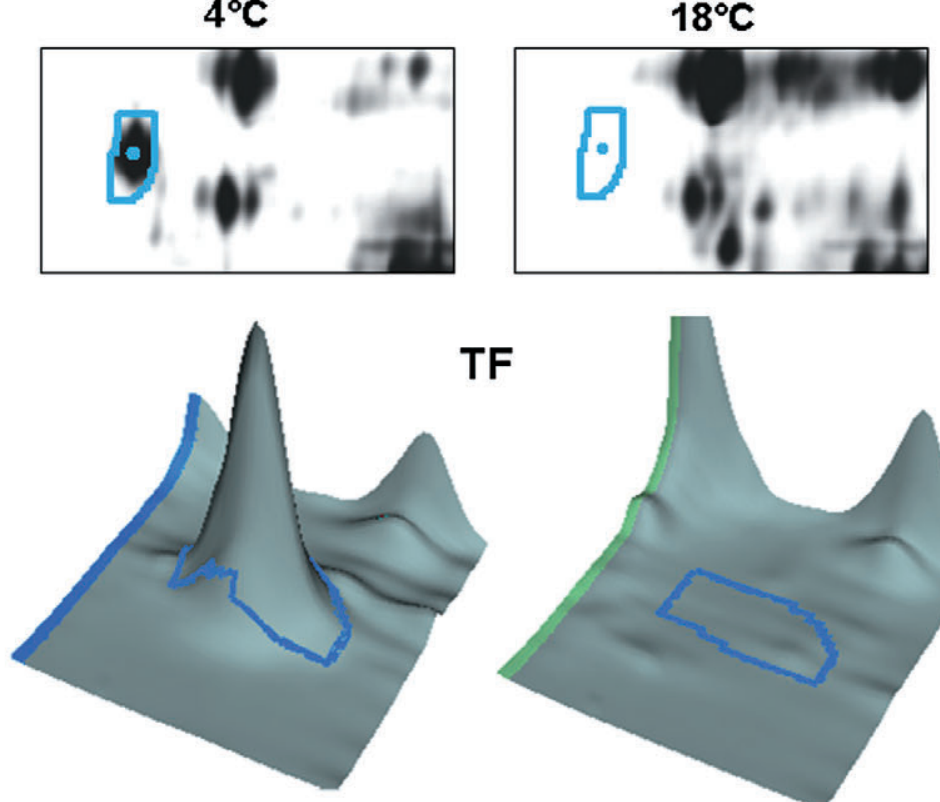

TF
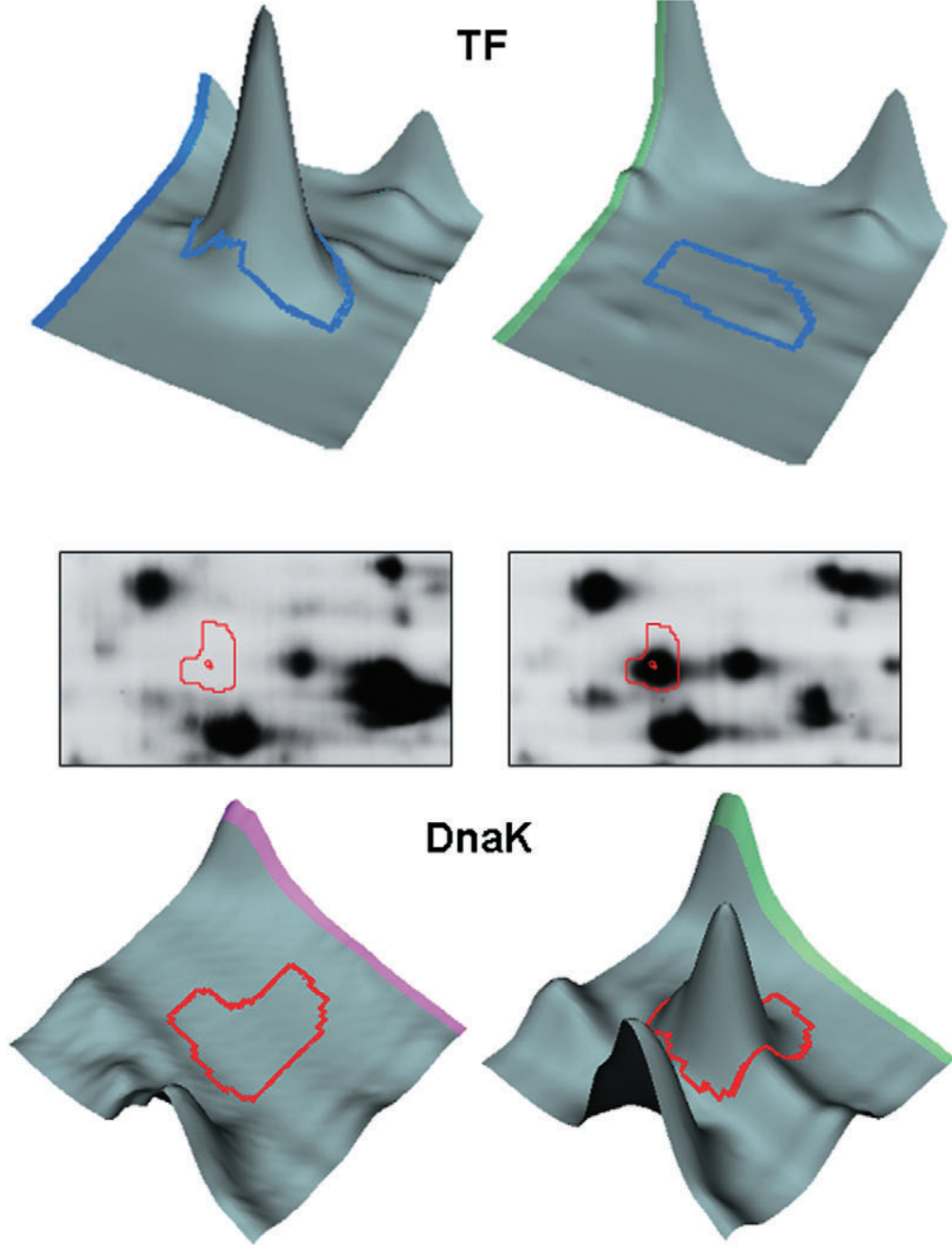

\section{nak}

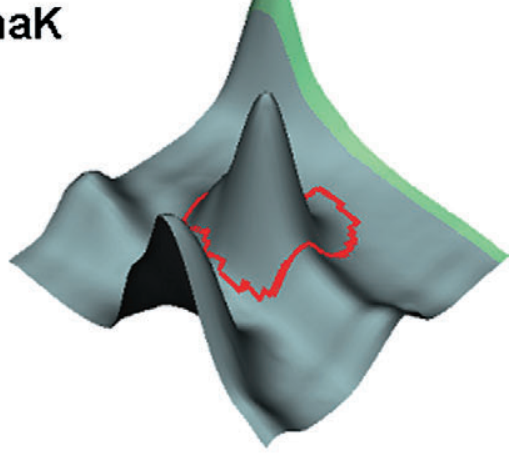

Fig. 5. Comparative analysis of spots containing the trigger factor TF and DnaK from Pseudoalteromonas haloplanktis TAC125 grown at $4^{\circ} \mathrm{C}$ (left panels) and $18^{\circ} \mathrm{C}$ (right panels). Spot views on 2D gels (circled) and three-dimensional images. Adapted by permission from John Wiley and Sons: Molecular Microbiology (Piette et al., 2010), copyright 2010 . ii. Under this imbalanced synthesis of folding assistants, TF obviously rescues the chaperone function and should be regarded as the primary chaperone for the growth of these strains in the cold (Piette et al., 2010). An interesting parallel can be drawn between hyperthermophilic Archaea (which only encode simplified chaperone systems in their genome) and those psychrophiles that only express a reduced set of chaperones. The significance of such a similarity deserves further investigation.

iii. It has been shown that the induced expression of HSPs in E. coli is harmful to cells at $4^{\circ} \mathrm{C}$ and that it reduces cell viability at this temperature (Kandror and
Goldberg, 1997). Accordingly, the observed cold repression of HSPs would be beneficial to these psychrophilic bacteria.

iv. When grown at low temperature, E. coli overexpresses the cold shock TF and represses the synthesis of HSPs (Kandror and Goldberg, 1997). Considering this regulation, it is tempting to propose that psychrophiles permanently make use of the cold stress response in mesophiles to ensure their sustained growth at low temperature.

In sharp contrast with the above mentioned pattern, almost the inverse regulation has been found in 


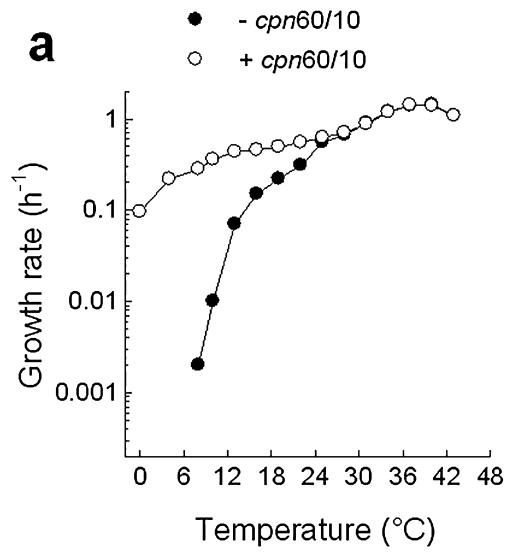

b

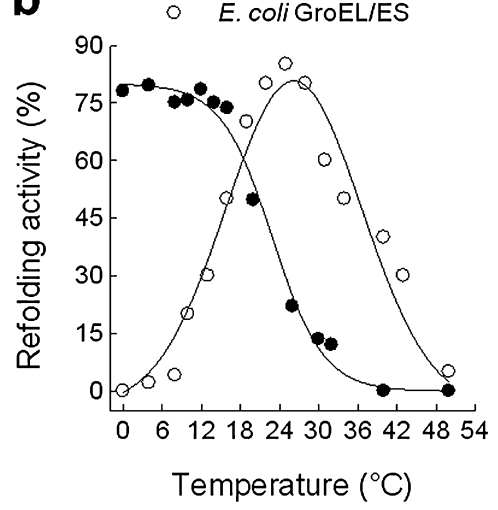

Fig. 6. In vivo and in vitro properties of the chaperonins of Oleispira antarctica.

A. Effect of expression of the $O$. antarctica chaperonins on the growth of $E$. coli at different temperatures.

B. In vitro refolding activities of $O$. antarctica Cpn60/10 and E. coli GroEL/ES chaperonins at different temperatures.

Reprinted by permission from Macmillan Publishers: Nature Biotechnology (Ferrer et al., 2003), copyright 2003.
$P$. arcticus, with upregulation of the GroEL/ES chaperonins and repression of TF in cold conditions (Zheng et al., 2007; Bergholz et al., 2009). Increased synthesis of chaperonins has also been reported in S. alaskensis (Ting et al., 2010). Such discrepancies are puzzling but are not an isolated example of the differences observed among the various psychrophilic microorganisms, as already mentioned. Evidently, increased synthesis of chaperonins would help the folding of large proteins, which are the preferred substrate of GroEL/ES, but repression of TF in $P$. arcticus remains to be properly explained. In any case, it is obvious that distinct strategies have been adopted by psychrophiles to assist proper protein folding. In this respect, a third alternative has been proposed for $S$. alaskensis (Ting et al., 2010). This bacterium possesses two sets of dnaK-dnaJ-grpE gene clusters (DnaK and its co-chaperones). Quantitative proteomics has suggested that one set functions as a low-temperature chaperone system whereas the other set functions at higher growth temperatures.

\section{Psychrophilic chaperonins expressed in E. coli}

One of the most significant and appealing observations in the field of psychrophilic protein folding has been the finding that expression of chaperonins from the bacterium Oleispira antarctica in E. coli allows the growth of this mesophilic bacterium at low temperature (Ferrer et al., 2003). As shown in Fig. 6A, E. coli fails to grow below $8^{\circ} \mathrm{C}$. However, expression of the GroEL/ES homologues from $O$. antarctica (termed Cpn60/10) has been shown to result in a 141 -fold faster growth at $8^{\circ} \mathrm{C}$, in a significant growth below $4^{\circ} \mathrm{C}$ and in a theoretical minimal temperature for growth at $-13.7^{\circ} \mathrm{C}$. This finding came as a real surprise for researchers involved in psychrophily because it demonstrated that only one protein complex is responsible for growth in the cold whereas it was generally assumed that tremendous cellular adaptations are required. This positive effect of $O$. antarctica chaperonins has been corre- lated to a cold-adapted refolding activity as illustrated in Fig. 6B. It has also been shown that this refolding activity originates from a double to single ring transition leading to an active heptameric chaperonin oligomer at $4-10^{\circ} \mathrm{C}$ (Ferrer et al., 2004). Taking advantage of these properties, the ArcticExpress E. coli cells from Stratagene (USA) have been engineered to coexpress the cold-active chaperonins with a recombinant protein of interest, therefore improving protein processing at low temperature and increasing the yield of active, soluble recombinant proteins.

But here again, a conflicting result has been reported for $P$. haloplanktis, another Antarctic bacterium. Indeed, expression of its chaperonins in $E$. coli failed to promote growth of the mesophilic bacterium at low temperature (Medigue et al., 2005). Furthermore, it has been shown that GroEL from $P$. haloplanktis is not cold-adapted: it is inefficient at low temperature as its ATPase activity is reduced to the same extent as that of its $E$. coli homologue (Tosco et al., 2003). We are therefore faced with drastically opposite properties of chaperonins from two Gram-negative Antarctic bacteria.

\section{Concluding remarks: is there a unified model?}

The various conflicting reports on protein folding in psychrophiles mentioned in this review will probably leave the reader rather sceptical. Although different methodologies or technical artefacts may have occasionally produced unreliable results, we believe that all these discrepancies mainly reflect the diverse strategies used by microorganisms to adapt to life in the cold. This hypothesis is based on a previous observation made by Bowman (2008). In a review of genome data from psychrophiles, he concluded that the lack of common features shared by these bacterial genomes suggests that cold adaptation superimposes on pre-existing cellular organization and, accordingly, that the adaptive strategies may differ between the various microorganisms. A similar conclusion can be reached via 
proteomic studies: proteins overexpressed at low temperature do not constitute a conserved set of proteins in terms of identification and expression level. Such heterogeneous upregulation supports the view that coldadaptation mechanisms are constrained by the speciesspecific cellular structure and organization, resulting in distinct adaptive strategies.

The occurrence of cold-adapted and non-cold-adapted chaperonins in psychrophiles also suggests that properties of the chaperone machinery reflect the microbial lifestyle. On the one hand, microorganisms thriving permanently in cold conditions (e.g. polar and deep-sea sediments, permafrost, etc.) would advantageously possess cold-adapted folding assistants. On the other hand, microorganisms subjected to seasonal or local temperature variations (e.g. melting sea ice, polar surface soils, etc.) would maintain a heat shock response involving non-coldadapted chaperones remaining active at transiently high temperatures. The emerging concept of duplicated gene sets, sharing the same function but differentially expressed at low or high temperatures (as noted for PPiases and DnaK gene clusters), also supports this view. It is expected that further investigations will clarify the still ambiguous status of protein folding adaptation in psychrophiles.

\section{Acknowledgements}

This work was supported by F.R.S.-FNRS (Fonds National de la Recherche Scientifique, Belgium, FRFC grants to G.F.). F.P. was supported by the European Space Agency (ExanamProdex Experiment Arrangement). The Institut Polaire Français Paul Emile Victor is also acknowledged for support at early stages of the work. F.P. was a FRIA research fellow and C.S. is a F.R.S.-FNRS research fellow.

\section{References}

Albanese, V., and Frydman, J. (2002) Where chaperones and nascent polypeptides meet. Nat Struct Biol 9: 716-718.

Allen, M.A., Lauro, F.M., Williams, T.J., Burg, D., Siddiqui, K.S., and De Francisci, D., et al. (2009) The genome sequence of the psychrophilic archaeon, Methanococcoides burtonii: the role of genome evolution in cold adaptation. ISME J 3: 1012-1035.

Ayala-del-Rio, H.L., Chain, P.S., Grzymski, J.J., Ponder, M.A., Ivanova, N., and Bergholz, P.W., et al. (2010) The genome sequence of Psychrobacter arcticus 273-4, a psychroactive Siberian permafrost bacterium, reveals mechanisms for adaptation to low-temperature growth. Appl Environ Microbiol 76: 2304-2312.

Bakermans, C., Tollaksen, S.L., Giometti, C.S., Wilkerson, C., Tiedje, J.M., and Thomashow, M.F. (2007) Proteomic analysis of Psychrobacter cryohalolentis K5 during growth at subzero temperatures. Extremophiles 11: 343-354.
Baldwin, R.L. (2008) The search for folding intermediates and the mechanism of protein folding. Annu Rev Biophys 37: $1-21$.

Bergholz, P.W., Bakermans, C., and Tiedje, J.M. (2009) Psychrobacter arcticus 273-4 uses resource efficiency and molecular motion adaptations for subzero temperature growth. J Bacteriol 191: 2340-2352.

Bowman, J.B. (2008) Genomic analysis of psychrophilic prokaryotes. In Psychrophiles, from Biodiversity to Biotechnology. Margesin, R., Schinner, F., Marx, J.C., and Gerday, C. (eds). Berlin, Heidelberg, Germany: Springer-Verlag, pp. 265-284.

Campanaro, S., Williams, T.J., Burg, D.W., De Francisci, D., Treu, L., Lauro, F.M., and Cavicchioli, R. (2011) Temperature-dependent global gene expression in the Antarctic archaeon Methanococcoides burtonii. Environ Microbiol (in press): doi:10.1111/j.1462-2920.2010.02367.x.

Cartier, G., Lorieux, F., Allemand, F., Dreyfus, M., and Bizebard, T. (2010) Cold adaptation in DEAD-box proteins. Biochemistry 49: 2636-2646.

Cary, S.C., McDonald, I.R., Barrett, J.E., and Cowan, D.A. (2010) On the rocks: the microbiology of Antarctic Dry Valley soils. Nat Rev Microbiol 8: 129-138.

Casanueva, A., Tuffin, M., Cary, C., and Cowan, D.A. (2010) Molecular adaptations to psychrophily: the impact of 'omic' technologies. Trends Microbiol 18: 374-381.

Cowan, D.A., Casanueva, A., and Stafford, W. (2007) Ecology and biodiversity of cold-adapted microorganisms. In Physiology and Biochemistry of Extremophiles. Gerday, C., and Glansdorff, N. (eds). Washington, DC, USA: ASM Press, pp. 119-132.

D'Amico, S., Collins, T., Marx, J.C., Feller, G., and Gerday, C. (2006) Psychrophilic microorganisms: challenges for life. EMBO Rep 7: 385-389.

Dalluge, J.J., Hamamoto, T., Horikoshi, K., Morita, R.Y., Stetter, K.O., and McCloskey, J.A. (1997) Posttranscriptional modification of tRNA in psychrophilic bacteria. J Bacteriol 179: 1918-1923.

Deming, J.W. (2002) Psychrophiles and polar regions. Curr Opin Microbiol 5: 301-309.

Duchaud, E., Boussaha, M., Loux, V., Bernardet, J.F., Michel, C., and Kerouault, B., et al. (2007) Complete genome sequence of the fish pathogen Flavobacterium psychrophiIum. Nat Biotechnol 25: 763-769.

Feller, G. (2010) Protein stability and enzyme activity at extreme biological temperatures. J Phys Condens Matter 22: 323101.

Feller, G., and Gerday, C. (2003) Psychrophilic enzymes: hot topics in cold adaptation. Nat Rev Microbiol 1: 200-208.

Ferrer, M., Chernikova, T.N., Yakimov, M.M., Golyshin, P.N., and Timmis, K.N. (2003) Chaperonins govern growth of Escherichia coli at low temperatures. Nat Biotechnol 21: 1266-1267.

Ferrer, M., Lunsdorf, H., Chernikova, T.N., Yakimov, M., Timmis, K.N., and Golyshin, P.N. (2004) Functional consequences of single:double ring transitions in chaperonins: life in the cold. Mol Microbiol 53: 167-182.

Friedmann, E.I. (1982) Endolithic microorganisms in the Antarctic cold desert. Science 215: 1045-1053.

Galtier, N., and Lobry, J.R. (1997) Relationships between genomic $\mathrm{G}+\mathrm{C}$ content, RNA secondary structures, and 
optimal growth temperature in prokaryotes. $J$ Mol Evol 44: 632-636.

Gerday, C., and Glansdorff, N. (2007) Physiology and Biochemistry of Extremophiles. Washington, DC, USA: ASM Press.

Gilichinsky, D., Rivkina, E., Bakermans, C., Shcherbakova, V., Petrovskaya, L., Ozerskaya, S., et al. (2005) Biodiversity of cryopegs in permafrost. FEMS Microbiol Ecol 53: 117-128.

Goodchild, A., Saunders, N.F., Ertan, H., Raftery, M., Guilhaus, M., Curmi, P.M., and Cavicchioli, R. (2004) A proteomic determination of cold adaptation in the Antarctic archaeon, Methanococcoides burtonii. Mol Microbiol 53: 309-321.

Goodchild, A., Raftery, M., Saunders, N.F., Guilhaus, M., and Cavicchioli, R. (2005) Cold adaptation of the Antarctic archaeon, Methanococcoides burtonii assessed by proteomics using ICAT. J Proteome Res 4: 473-480.

Hartl, F.U., and Hayer-Hartl, M. (2009) Converging concepts of protein folding in vitro and in vivo. Nat Struct Mol Biol 16: 574-581.

Jenkins, J., and Pickersgill, R. (2001) The architecture of parallel $\beta$-helices and related folds. Prog Biophys $\mathrm{Mol}$ Biol 77: 111-175.

Kandror, O., and Goldberg, A.L. (1997) Trigger factor is induced upon cold shock and enhances viability of Escherichia coli at low temperatures. Proc Natl Acad Sci USA 94: 4978-4981.

Kawamoto, J., Kurihara, T., Kitagawa, M., Kato, I., and Esaki, N. (2007) Proteomic studies of an Antarctic cold-adapted bacterium, Shewanella livingstonensis Ac10, for global identification of cold-inducible proteins. Extremophiles 11: 819-826.

Khachane, A.N., Timmis, K.N., and dos Santos, V.A. (2005) Uracil content of 16S rRNA of thermophilic and psychrophilic prokaryotes correlates inversely with their optimal growth temperatures. Nucleic Acids Res 33: 40164022.

King, J., Haase-Pettingell, C., Robinson, A.S., Speed, M., and Mitraki, A. (1996) Thermolabile folding intermediates: inclusion body precursors and chaperonin substrates. FASEB J 10: 57-66.

Kramers, H.A. (1940) Brownian motion in a field of force and the diffusion model of chemical reactions. Physica 7: 284304.

Laksanalamai, P., Whitehead, T.A., and Robb, F.T. (2004) Minimal protein-folding systems in hyperthermophilic archaea. Nat Rev Microbiol 2: 315-324.

Lim, J., Thomas, T., and Cavicchioli, R. (2000) Low temperature regulated DEAD-box RNA helicase from the Antarctic archaeon, Methanococcoides burtonii. J Mol Biol 297: 553-567.

Margesin, R., Schinner, F., Marx, J.C., and Gerday, C. (2008) Psychrophiles, from Biodiversity to Biotechnology. Berlin, Heidelberg, Germany: Springer-Verlag.

Medigue, C., Krin, E., Pascal, G., Barbe, V., Bernsel, A., Bertin, P.N., et al. (2005) Coping with cold: the genome of the versatile marine Antarctica bacterium Pseudoalteromonas haloplanktis TAC125. Genome Res 15: 1325-1335.

Methe, B.A., Nelson, K.E., Deming, J.W., Momen, B., Melamud, E., Zhang, X., et al. (2005) The psychrophilic lifestyle as revealed by the genome sequence of Colwellia psychrerythraea $34 \mathrm{H}$ through genomic and proteomic analyses. Proc Natl Acad Sci USA 102: 10913-10918.

Piette, F., D'Amico, S., Struvay, C., Mazzucchelli, G., Renaut, J., Tutino, M.L., et al. (2010) Proteomics of life at low temperatures: trigger factor is the primary chaperone in the Antarctic bacterium Pseudoalteromonas haloplanktis TAC125. Mol Microbiol 76: 120-132.

Qiu, Y., Kathariou, S., and Lubman, D.M. (2006) Proteomic analysis of cold adaptation in a Siberian permafrost bacterium Exiguobacterium sibiricum 255-15 by twodimensional liquid separation coupled with mass spectrometry. Proteomics 6: 5221-5233.

Rabus, R., Ruepp, A., Frickey, T., Rattei, T., Fartmann, B., Stark, M., et al. (2004) The genome of Desulfotalea psychrophila, a sulfate-reducing bacterium from permanently cold Arctic sediments. Environ Microbiol 6: 887-902.

Riley, M., Staley, J.T., Danchin, A., Wang, T.Z., Brettin, T.S., Hauser, L.J., et al. (2008) Genomics of an extreme psychrophile, Psychromonas ingrahamii. BMC Genomics 9: 210. doi:210.1186/1471-2164-1189-1210.

Rodrigues, D.F., and Tiedje, J.M. (2008) Coping with our cold planet. Appl Environ Microbiol 74: 1677-1686.

Rodrigues, D.F., Ivanova, N., He, Z., Huebner, M., Zhou, J., and Tiedje, J.M. (2008) Architecture of thermal adaptation in an Exiguobacterium sibiricum strain isolated from 3 million year old permafrost: a genome and transcriptome approach. BMC Genomics 9: 547. doi:510.1186/14712164-1189-1547.

Russell, N.J. (2007) Psychrophiles: membrane adaptations. In Physiology and Biochemistry of Extremophiles. Gerday, C., and Glansdorff, N. (eds). Washington, DC, USA: ASM Press, pp. 155-164.

Saunders, N.F., Thomas, T., Curmi, P.M., Mattick, J.S., Kuczek, E., Slade, R., et al. (2003) Mechanisms of thermal adaptation revealed from the genomes of the Antarctic Archaea Methanogenium frigidum and Methanococcoides burtonii. Genome Res 13: 1580-1588.

Scholz, C., Stoller, G., Zarnt, T., Fischer, G., and Schmid, F.X. (1997) Cooperation of enzymatic and chaperone functions of trigger factor in the catalysis of protein folding. EMBO $J$ 16: 54-58.

Siddiqui, K.S., and Cavicchioli, R. (2006) Cold-adapted enzymes. Annu Rev Biochem 75: 403-433.

Smalas, A.O., Leiros, H.K., Os, V., and Willassen, N.P. (2000) Cold adapted enzymes. Biotechnol Annu Rev 6: 1-57.

Stoller, G., Rucknagel, K.P., Nierhaus, K.H., Schmid, F.X., Fischer, G., and Rahfeld, J.U. (1995) A ribosomeassociated peptidyl-prolyl cis/trans isomerase identified as the trigger factor. EMBO J 14: 4939-4948.

Suzuki, Y., Haruki, M., Takano, K., Morikawa, M., and Kanaya, S. (2004) Possible involvement of an FKBP family member protein from a psychrotrophic bacterium Shewanella sp. SIB1 in cold-adaptation. Eur J Biochem 271: 1372-1381.

Ting, L., Williams, T.J., Cowley, M.J., Lauro, F.M., Guilhaus, M., Raftery, M.J., and Cavicchioli, R. (2010) Cold adaptation in the marine bacterium, Sphingopyxis alaskensis, assessed using quantitative proteomics. Environ Microbiol 12: 2658-2676.

Tosco, A., Birolo, L., Madonna, S., Lolli, G., Sannia, G., and 
Marino, G. (2003) GroEL from the psychrophilic bacterium Pseudoalteromonas haloplanktis TAC 125: molecular characterization and gene cloning. Extremophiles 7: 17-28.

Williams, T.J., Burg, D.W., Raftery, M.J., Poljak, A., Guilhaus, M., Pilak, O., and Cavicchioli, R. (2010) Global proteomic analysis of the insoluble, soluble, and supernatant fractions of the psychrophilic archaeon Methanococcoides burtonii. Part I: the effect of growth temperature. J Proteome Res 9: 640-652.
Zheng, S., Ponder, M.A., Shih, J.Y., Tiedje, J.M., Thomashow, M.F., and Lubman, D.M. (2007) A proteomic analysis of Psychrobacter articus 273-4 adaptation to low temperature and salinity using a 2-D liquid mapping approach. Electrophoresis 28: 467-488.

Zoldak, G., Aumuller, T., Lucke, C., Hritz, J., Oostenbrink, C., Fischer, G., and Schmid, F.X. (2009) A library of fluorescent peptides for exploring the substrate specificities of prolyl isomerases. Biochemistry 48: 10423-10436. 